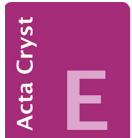
COMMUNICATIONS

ISSN 2056-9890

\section{Crystal structure of benzobicyclon}

\section{Gihaeng Kang, Jineun Kim, ${ }^{*}$ Hansu Lim and Tae Ho Kim*}

Department of Chemistry and Research Institute of Natural Sciences, Gyeongsang National University, Jinju 52828, Republic of Korea. *Correspondence e-mail:

thkim@gnu.ac.kr, jekim@gnu.ac.kr

Received 27 November 2015; accepted 2 December 2015

Edited by J. Simpson, University of Otago, New Zealand

In the title compound, $\mathrm{C}_{22} \mathrm{H}_{19} \mathrm{ClO}_{4} \mathrm{~S}_{2}$ [systematic name: 3-(2chloro-4-mesylbenzoyl)-4-(phenylsulfanyl)bicyclo[3.2.1]oct-3en-2-one], which is an unclassified herbicide, the dihedral angle between the plane of the phenyl and chlorobenzene rings is $19.9(2)^{\circ}$. In the crystal, $\mathrm{C}-\mathrm{H} \cdots \mathrm{O}$ hydrogen bonds link adjacent molecules, generating two-dimensional networks extending parellel to (011).

Keywords: crystal structure; benzobicyclon; bicyclo[3.2.1]oct-2-en-4-one; herbicide.

CCDC reference: 1440215

\section{Related literature}

For information on the herbicidal properties of the title compound, see: Im et al. (2015). For related crystal structures, see: Brown et al. (2007); Hou et al. (2010).

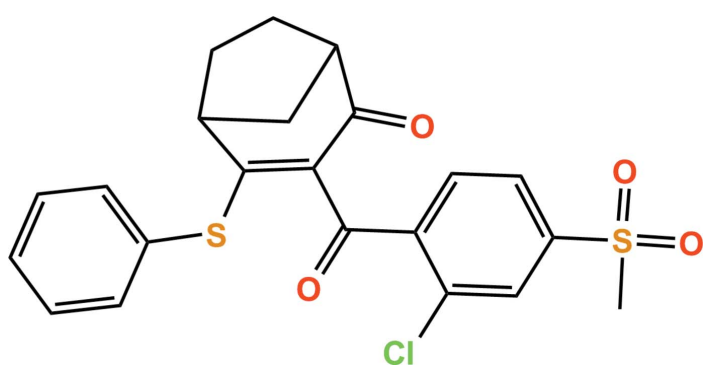

\section{Experimental}

\subsection{Crystal data}

\section{$\mathrm{C}_{22} \mathrm{H}_{19} \mathrm{ClO}_{4} \mathrm{~S}_{2}$}

$M_{r}=446.94$

$$
\begin{aligned}
& b=10.7218(6) \AA \\
& c=12.0169(6) \AA \\
& \beta=91.449(3)^{\circ} \\
& V=1997.85(18) \AA^{3} \\
& Z=4
\end{aligned}
$$

\subsection{Data collection \\ Bruker APEXII CCD diffractometer \\ Absorption correction: multi-scan (SADABS; Bruker, 2014) \\ $T_{\min }=0.687, T_{\max }=0.746$}

\subsection{Refinement}

$R\left[F^{2}>2 \sigma\left(F^{2}\right)\right]=0.053$

$w R\left(F^{2}\right)=0.142$

$S=1.07$

3504 reflections

263 parameters

$\mathrm{H}$-atom parameters constrained

$\Delta \rho_{\max }=0.97 \mathrm{e} \AA^{-3}$

$\Delta \rho_{\min }=-0.54{\mathrm{e} \AA^{-3}}^{-3}$

Table 1

Hydrogen-bond geometry $\left(\AA{ }^{\circ}\right)$.

\begin{tabular}{lllll}
\hline$D-\mathrm{H} \cdots A$ & $D-\mathrm{H}$ & $\mathrm{H} \cdots A$ & $D \cdots A$ & $D-\mathrm{H} \cdots A$ \\
\hline $\mathrm{C} 1-\mathrm{H} 1 B \cdots \mathrm{O} 4^{\mathrm{i}}$ & 0.98 & 2.44 & $3.322(4)$ & 150 \\
$\mathrm{C} 1-\mathrm{H} 1 C \cdots \mathrm{O} 4^{\mathrm{ii}}$ & 0.98 & 2.52 & $3.477(5)$ & 165 \\
$\mathrm{C} 3-\mathrm{H} 3 \cdots \mathrm{O} 2^{\mathrm{iii}}$ & 0.95 & 2.53 & $3.422(4)$ & 157 \\
\hline
\end{tabular}

Symmetry codes: (i) $x,-y+\frac{1}{2}, z+\frac{1}{2}$; (ii) $-x, y-\frac{1}{2},-z+\frac{3}{2}$; (iii) $-x,-y+1,-z+2$.

Data collection: APEX2 (Bruker, 2014); cell refinement: SAINT (Bruker, 2014); data reduction: $S A I N T$; $\operatorname{program}(\mathrm{s})$ used to solve structure: SHELXS97 (Sheldrick, 2008); program(s) used to refine structure: SHELXL2014 (Sheldrick, 2015); molecular graphics: DIAMOND (Brandenburg, 2010); software used to prepare material for publication: SHELXTL (Sheldrick, 2008).

\title{
Acknowledgements
}

This research was supported by the Basic Science Research Program through the National Research Foundation of Korea (NRF) funded by the Ministry of Education, Science and Technology (No. 2015R1D1A4A01020317).

Supporting information for this paper is available from the IUCr electronic archives (Reference: SJ5485).

\section{References}

Brandenburg, K. (2010). DIAMOND. Crystal Impact GbR, Bonn, Germany. Brown, P. M., Käppel, N., Murphy, P. J., Coles, S. J. \& Hursthouse, M. B. (2007). Tetrahedron, 63, 1100-1106.

Bruker (2014). APEX2, SAINT and SADABS. Bruker AXS Inc., Madison, Wisconsin, USA.

Hou, Y.-J., Chu, W.-Y., Sui, J. \& Sun, Z.-Z. (2010). Z. Kristallogr. 225, 465-466. Im, S. J., Abd El-Aty, A. M., Lee, Y.-J., Rahman, M. M., Kim, S.-W., Choi, J.-H. \& Shim, J.-H. (2015). Food Chem. 168, 404-409.

Sheldrick, G. M. (2008). Acta Cryst. A64, 112-122.

Sheldrick, G. M. (2015). Acta Cryst. C71, 3-8. 


\section{supporting information}

Acta Cryst. (2015). E71, o1035 [https://doi.org/10.1107/S2056989015023221]

\section{Crystal structure of benzobicyclon}

\section{Gihaeng Kang, Jineun Kim, Hansu Lim and Tae Ho Kim}

\section{S1. Comment}

Benzobicyclon is a newly developed compound with potent herbicidal activity against Scirpus juncoides, one of the most troublesome weeds in paddy fields. (Im et al., 2015). However, until now its crystal structure has not been reported. In the title compound (Fig. 1), the dihedral angle between the plane of the phenyl and chlorobenzene rings is 19.9 (2) ${ }^{\circ}$. All bond lengths and bond angles are normal and comparable to those observed in the crystal structures of similar compounds (Brown et al., 2007; Hou et al., 2010).

In the crystal structure (Fig. 2), intermolecular $\mathrm{C} 1-\mathrm{H} 1 \mathrm{~B} \cdots \mathrm{O} 4$ hydrogen bond link adjacent molecules, forming chains along the $c$-axis direction. These chains are linked by $\mathrm{C} 1-\mathrm{H} 1 \mathrm{C} \cdots \mathrm{O} 4$ and $\mathrm{C} 3-\mathrm{H} 3 \cdots \mathrm{O} 2$ hydrogen bonds (Table 1), resulting in two-dimensional networks extending parellel to the (011) plane.

\section{S2. Experimental}

The title compound was purchased from the Dr. Ehrenstorfer $\mathrm{GmbH}$ Company. Slow evaporation of a solution in $\mathrm{CH}_{2} \mathrm{Cl}_{2}$ gave single crystals suitable for X-ray analysis.

\section{S3. Refinement}

All H-atoms were positioned geometrically and refined using a riding model with $\mathrm{d}(\mathrm{C}-\mathrm{H})=1.00 \AA, U_{\text {iso }}=1.2 U_{\text {eq }}(\mathrm{C})$ for $\mathrm{Csp}^{3}-\mathrm{H}, \mathrm{d}(\mathrm{C}-\mathrm{H})=0.99 \AA, U_{\text {iso }}=1.2 U_{\text {eq }}(\mathrm{C})$ for $\mathrm{CH}_{2}$ groups, $\mathrm{d}(\mathrm{C}-\mathrm{H})=0.98 \AA, U_{\text {iso }}=1.2 U_{\text {eq }}(\mathrm{C})$ for $\mathrm{CH}_{3}$ groups, $\mathrm{d}(\mathrm{C}-$ $\mathrm{H})=0.95 \AA, U_{\text {iso }}=1.2 U_{\text {eq }}(\mathrm{C})$ for aromatic $\mathrm{C}-\mathrm{H}$.

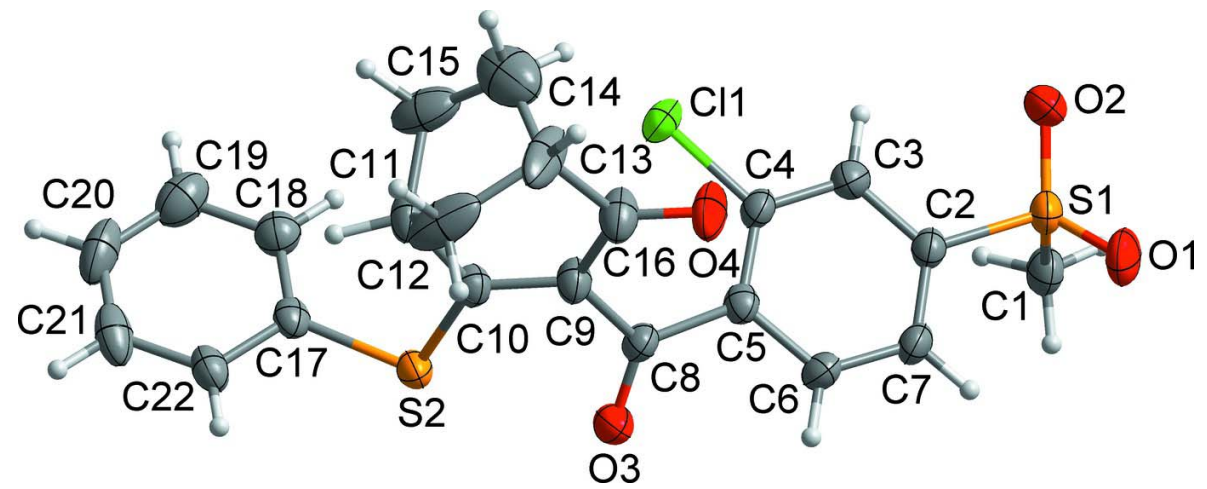

Figure 1

The asymmetric unit of the title compound with the atom-numbering scheme. Displacement ellipsoids are drawn at the $50 \%$ probability level. $\mathrm{H}$ atoms are shown as small spheres of arbitrary radius. 


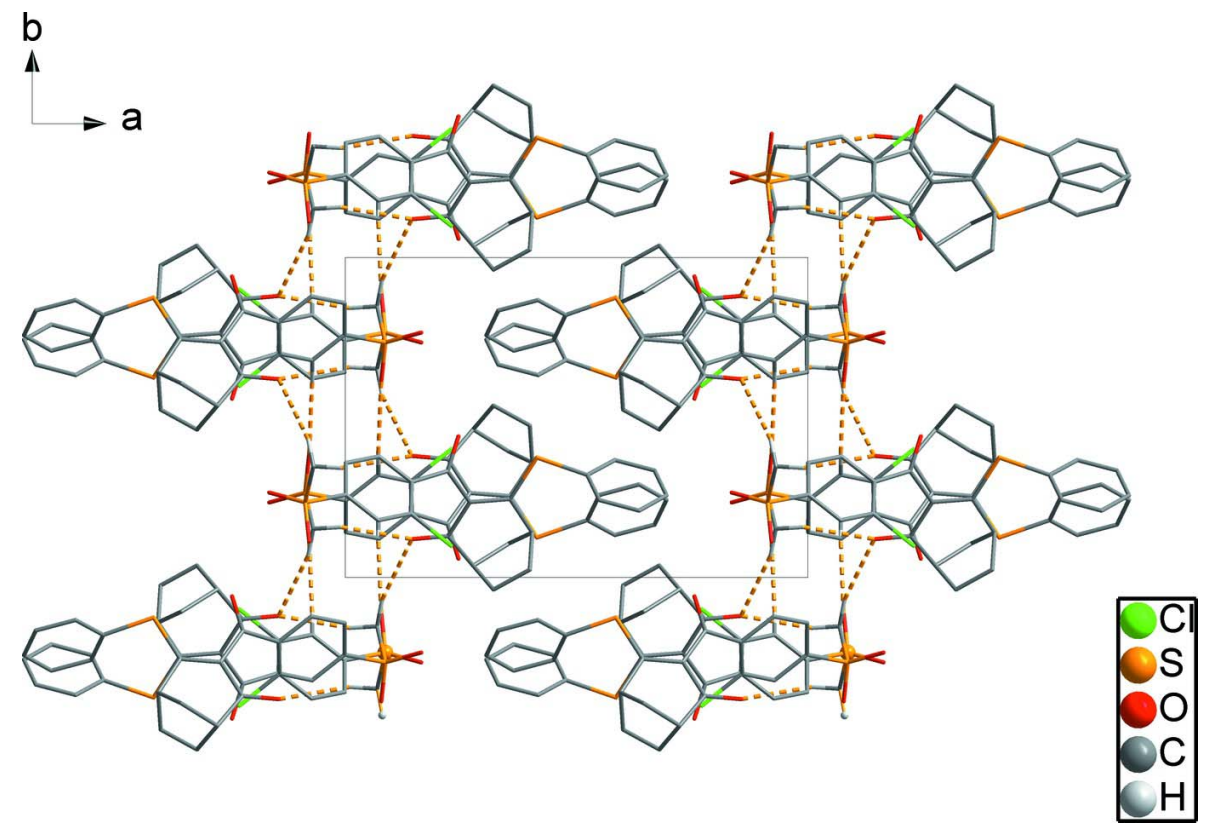

Figure 2

Crystal packing viewed along the $c$ axis. The intermolecular hydrogen bonds are shown as dashed lines.

\section{3-(2-Chloro-4-mesylbenzoyl)-4-(phenylsulfanyl)bicyclo[3.2.1]oct-3-en-2-one}

\section{Crystal data}

$\mathrm{C}_{22} \mathrm{H}_{19} \mathrm{ClO}_{4} \mathrm{~S}_{2}$

$M_{r}=446.94$

Monoclinic, $P 2_{1} / c$

$a=15.5111(8) \AA$

$b=10.7218(6) \AA$

$c=12.0169(6) \AA$

$\beta=91.449(3)^{\circ}$

$V=1997.85(18) \AA^{3}$

$Z=4$

\section{Data collection}

Bruker APEXII CCD diffractometer

$\varphi$ and $\omega$ scans

Absorption correction: multi-scan

(SADABS; Bruker, 2014)

$T_{\min }=0.687, T_{\max }=0.746$

15850 measured reflections

\section{Refinement}

Refinement on $F^{2}$

Least-squares matrix: full

$R\left[F^{2}>2 \sigma\left(F^{2}\right)\right]=0.053$

$w R\left(F^{2}\right)=0.142$

$S=1.07$

3504 reflections

263 parameters

0 restraints
$F(000)=928$

$D_{\mathrm{x}}=1.486 \mathrm{Mg} \mathrm{m}^{-3}$

Mo $K \alpha$ radiation, $\lambda=0.71073 \AA$

Cell parameters from 2645 reflections

$\theta=2.6-24.7^{\circ}$

$\mu=0.43 \mathrm{~mm}^{-1}$

$T=173 \mathrm{~K}$

Block, colourless

$0.15 \times 0.11 \times 0.06 \mathrm{~mm}$

3504 independent reflections

2584 reflections with $I>2 \sigma(I)$

$R_{\text {int }}=0.055$

$\theta_{\text {max }}=25.0^{\circ}, \theta_{\text {min }}=2.3^{\circ}$

$h=-18 \rightarrow 18$

$k=-11 \rightarrow 12$

$l=-14 \rightarrow 12$

Hydrogen site location: inferred from neighbouring sites

$\mathrm{H}$-atom parameters constrained

$w=1 /\left[\sigma^{2}\left(F_{\mathrm{o}}^{2}\right)+(0.0619 P)^{2}+2.0045 P\right]$

where $P=\left(F_{\mathrm{o}}^{2}+2 F_{\mathrm{c}}^{2}\right) / 3$

$(\Delta / \sigma)_{\max }<0.001$

$\Delta \rho_{\max }=0.97 \mathrm{e} \AA^{-3}$

$\Delta \rho_{\min }=-0.54 \mathrm{e} \AA^{-3}$ 


\section{Special details}

Geometry. All esds (except the esd in the dihedral angle between two 1.s. planes) are estimated using the full covariance matrix. The cell esds are taken into account individually in the estimation of esds in distances, angles and torsion angles; correlations between esds in cell parameters are only used when they are defined by crystal symmetry. An approximate (isotropic) treatment of cell esds is used for estimating esds involving l.s. planes.

Fractional atomic coordinates and isotropic or equivalent isotropic displacement parameters $\left(\hat{A}^{2}\right)$

\begin{tabular}{|c|c|c|c|c|}
\hline & $x$ & $y$ & $z$ & $U_{\text {iso }} * / U_{\text {eq }}$ \\
\hline $\mathrm{Cl1}$ & $0.22882(6)$ & $0.40049(9)$ & $0.83242(9)$ & $0.0464(3)$ \\
\hline S1 & $-0.08667(6)$ & $0.26508(9)$ & $0.95779(9)$ & $0.0361(3)$ \\
\hline S2 & $0.41445(6)$ & $0.12501(8)$ & $0.64532(8)$ & $0.0324(3)$ \\
\hline $\mathrm{O} 1$ & $-0.16416(16)$ & $0.2346(3)$ & $0.8963(2)$ & $0.0495(8)$ \\
\hline $\mathrm{O} 2$ & $-0.07793(17)$ & $0.3867(2)$ & $1.0065(3)$ & $0.0499(8)$ \\
\hline $\mathrm{O} 3$ & $0.24478(16)$ & $0.0592(2)$ & $0.6713(2)$ & $0.0429(7)$ \\
\hline $\mathrm{O} 4$ & $0.14394(16)$ & $0.3801(3)$ & $0.5647(3)$ & $0.0479(8)$ \\
\hline $\mathrm{C} 1$ & $-0.0691(2)$ & $0.1524(4)$ & $1.0616(3)$ & $0.0401(10)$ \\
\hline H1A & -0.1083 & 0.1674 & 1.1228 & $0.060^{*}$ \\
\hline H1B & -0.0093 & 0.1576 & 1.0895 & $0.060^{*}$ \\
\hline $\mathrm{H} 1 \mathrm{C}$ & -0.0799 & 0.0692 & 1.0304 & $0.060^{*}$ \\
\hline $\mathrm{C} 2$ & $0.0015(2)$ & $0.2413(3)$ & $0.8691(3)$ & $0.0303(8)$ \\
\hline $\mathrm{C} 3$ & $0.0716(2)$ & $0.3211(3)$ & $0.8792(3)$ & $0.0339(9)$ \\
\hline $\mathrm{H} 3$ & 0.0715 & 0.3884 & 0.9305 & $0.041 *$ \\
\hline $\mathrm{C} 4$ & $0.1417(2)$ & $0.3002(3)$ & $0.8126(3)$ & $0.0309(8)$ \\
\hline $\mathrm{C} 5$ & $0.1442(2)$ & $0.2011(3)$ & $0.7389(3)$ & $0.0290(8)$ \\
\hline C6 & $0.0730(2)$ & $0.1218(3)$ & 0.7314 (3) & $0.0335(9)$ \\
\hline H6 & 0.0736 & 0.0530 & 0.6816 & $0.040^{*}$ \\
\hline $\mathrm{C} 7$ & $0.0013(2)$ & $0.1420(3)$ & $0.7954(3)$ & $0.0338(9)$ \\
\hline H7 & -0.0473 & 0.0883 & 0.7890 & $0.041^{*}$ \\
\hline $\mathrm{C} 8$ & $0.2214(2)$ & $0.1671(3)$ & $0.6715(3)$ & $0.0302(8)$ \\
\hline C9 & $0.2696(2)$ & $0.2642(3)$ & $0.6103(3)$ & $0.0336(9)$ \\
\hline $\mathrm{C} 10$ & $0.3565(2)$ & $0.2546(3)$ & $0.5982(3)$ & $0.0310(8)$ \\
\hline C11 & $0.4045(2)$ & 0.3595 (4) & $0.5419(3)$ & $0.0382(9)$ \\
\hline H11 & 0.4656 & 0.3361 & 0.5274 & $0.046^{*}$ \\
\hline C12 & $0.3542(4)$ & $0.3935(5)$ & $0.4330(3)$ & $0.0668(16)$ \\
\hline $\mathrm{H} 12 \mathrm{~A}$ & 0.3347 & 0.3191 & 0.3906 & $0.080^{*}$ \\
\hline H12B & 0.3870 & 0.4499 & 0.3845 & $0.080^{*}$ \\
\hline C13 & $0.2767(3)$ & $0.4631(4)$ & $0.4939(5)$ & $0.0711(17)$ \\
\hline H13 & 0.2407 & 0.5144 & 0.4411 & $0.085^{*}$ \\
\hline C14 & 0.3207 (4) & $0.5376(5)$ & $0.5754(5)$ & 0.0755 (17) \\
\hline H14A & 0.2838 & 0.5499 & 0.6405 & $0.091^{*}$ \\
\hline H14B & 0.3341 & 0.6204 & 0.5438 & $0.091^{*}$ \\
\hline $\mathrm{C} 15$ & $0.3992(3)$ & 0.4753 (4) & $0.6092(4)$ & $0.0622(14)$ \\
\hline $\mathrm{H} 15 \mathrm{~A}$ & 0.3983 & 0.4548 & 0.6895 & $0.075^{*}$ \\
\hline H15B & 0.4495 & 0.5295 & 0.5958 & $0.075^{*}$ \\
\hline $\mathrm{C} 16$ & $0.2224(2)$ & $0.3702(4)$ & $0.5594(4)$ & $0.0466(11)$ \\
\hline C17 & $0.5234(2)$ & 0.1795 (3) & $0.6555(3)$ & $0.0299(8)$ \\
\hline $\mathrm{C} 18$ & $0.5496(3)$ & 0.2579 (3) & $0.7411(3)$ & $0.0404(10)$ \\
\hline
\end{tabular}




\begin{tabular}{lllll} 
H18 & 0.5085 & 0.2892 & 0.7914 & $0.048^{*}$ \\
C19 & $0.6355(3)$ & $0.2910(4)$ & $0.7538(4)$ & $0.0532(12)$ \\
H19 & 0.6536 & 0.3460 & 0.8118 & $0.064^{*}$ \\
C20 & $0.6948(3)$ & $0.2430(4)$ & $0.6807(4)$ & $0.0566(13)$ \\
H20 & 0.7540 & 0.2643 & 0.6896 & $0.068^{*}$ \\
C21 & $0.6690(3)$ & $0.1654(4)$ & $0.5962(4)$ & $0.0488(12)$ \\
H21 & 0.7103 & 0.1336 & 0.5465 & $0.059^{*}$ \\
C22 & $0.5831(2)$ & $0.1329(3)$ & $0.5825(3)$ & $0.0374(9)$ \\
H22 & 0.5652 & 0.0791 & 0.5236 & $0.045^{*}$ \\
\hline
\end{tabular}

Atomic displacement parameters $\left(\AA^{2}\right)$

\begin{tabular}{|c|c|c|c|c|c|c|}
\hline & $U^{11}$ & $U^{22}$ & $U^{33}$ & $U^{12}$ & $U^{13}$ & $U^{23}$ \\
\hline $\mathrm{Cl1}$ & $0.0325(5)$ & $0.0437(6)$ & $0.0632(7)$ & $-0.0172(4)$ & $0.0084(5)$ & $-0.0109(5)$ \\
\hline $\mathrm{S} 1$ & $0.0220(5)$ & $0.0359(5)$ & $0.0509(6)$ & $-0.0007(4)$ & 0.0085 & $-0.0012(4)$ \\
\hline $\mathrm{S} 2$ & $0.0273(5)$ & $0.0273(5)$ & $0.0430(6)$ & $0.0021(4)$ & $0.0086(4)$ & $0.0007(4)$ \\
\hline $\mathrm{O} 1$ & $0.0215(13)$ & 0.0641 (19) & $0.063(2)$ & $0.0002(12)$ & $-0.0001(13)$ & $-0.0002(15)$ \\
\hline $\mathrm{O} 2$ & $0.0421(16)$ & $0.0354(15)$ & $0.073(2)$ & $-0.0006(12)$ & $0.0196(15)$ & $-0.0119(14)$ \\
\hline $\mathrm{O} 3$ & $0.0372(15)$ & $0.0298(14)$ & $0.0624(19)$ & $-0.0013(12)$ & $0.0170(14)$ & $-0.0004(13)$ \\
\hline $\mathrm{O} 4$ & $0.0221(14)$ & $0.0543(17)$ & $0.067(2)$ & $0.0025(12)$ & $0.0014(13)$ & $0.0214(15)$ \\
\hline $\mathrm{C} 1$ & $0.030(2)$ & $0.046(2)$ & $0.045(2)$ & $-0.0030(17)$ & $0.0105(18)$ & 0.0022 (19) \\
\hline $\mathrm{C} 2$ & $0.0229(18)$ & $0.0305(18)$ & $0.038(2)$ & $-0.0010(14)$ & $0.0032(16)$ & $0.0033(16)$ \\
\hline $\mathrm{C} 3$ & 0.0296 (19) & $0.0289(18)$ & $0.043(2)$ & $-0.0007(15)$ & $0.0015(17)$ & $-0.0017(17)$ \\
\hline $\mathrm{C} 4$ & $0.0235(17)$ & $0.0294(18)$ & $0.040(2)$ & $-0.0053(15)$ & $0.0033(16)$ & $0.0032(16)$ \\
\hline $\mathrm{C} 5$ & $0.0254(18)$ & $0.0278(18)$ & $0.034(2)$ & $0.0000(14)$ & $0.0047(16)$ & $0.0064(16)$ \\
\hline C6 & $0.032(2)$ & 0.0277 (18) & $0.041(2)$ & $-0.0078(15)$ & $0.0045(17)$ & $-0.0026(17)$ \\
\hline $\mathrm{C} 7$ & $0.0230(18)$ & $0.0325(19)$ & $0.046(2)$ & $-0.0066(15)$ & $0.0019(17)$ & $0.0022(17)$ \\
\hline $\mathrm{C} 8$ & $0.0258(18)$ & $0.0282(19)$ & $0.037(2)$ & $-0.0045(15)$ & $0.0044(16)$ & $0.0028(16)$ \\
\hline $\mathrm{C} 9$ & $0.0239(18)$ & $0.036(2)$ & $0.041(2)$ & $0.0005(15)$ & $0.0052(16)$ & $0.0093(17)$ \\
\hline $\mathrm{C} 10$ & $0.0284(19)$ & 0.0329 (19) & $0.032(2)$ & $0.0003(15)$ & $0.0050(16)$ & $0.0034(16)$ \\
\hline $\mathrm{C} 11$ & $0.0225(18)$ & $0.044(2)$ & 0.049 (2) & $0.0000(16)$ & 0.0069 (17) & 0.0151 (19) \\
\hline $\mathrm{C} 12$ & 0.107 (4) & $0.068(3)$ & $0.026(2)$ & $-0.048(3)$ & $0.012(3)$ & $0.002(2)$ \\
\hline $\mathrm{C} 13$ & $0.026(2)$ & $0.044(3)$ & $0.141(5)$ & 0.0005 (19) & $-0.023(3)$ & $0.043(3)$ \\
\hline $\mathrm{C} 14$ & $0.095(4)$ & $0.061(3)$ & $0.072(4)$ & $0.008(3)$ & $0.031(3)$ & $0.018(3)$ \\
\hline $\mathrm{C} 15$ & $0.094(4)$ & $0.047(3)$ & $0.046(3)$ & -0.030 & $0.011(3)$ & $0.000(2)$ \\
\hline $\mathrm{C} 16$ & $0.029(2)$ & $0.048(2)$ & $0.063(3)$ & $0.0016(18)$ & $0.005(2)$ & $0.020(2)$ \\
\hline $\mathrm{C} 17$ & $0.0255(18)$ & 0.0270 & $0.037(2)$ & 0.0048 (14) & 0.0025 & $0.0080(16)$ \\
\hline C18 & $0.040(2)$ & $0.037(2)$ & $0.044(2)$ & $0.0001(17)$ & 0.0027 (19) & $0.0038(19)$ \\
\hline C19 & $0.049(3)$ & $0.055(3)$ & $0.055(3)$ & $-0.011(2)$ & $-0.012(2)$ & $0.009(2)$ \\
\hline $\mathrm{C} 20$ & $0.027(2)$ & $0.068(3)$ & $0.074(3)$ & $-0.009(2)$ & $-0.007(2)$ & $0.029(3)$ \\
\hline $\mathrm{C} 21$ & $0.033(2)$ & $0.056(3)$ & $0.058(3)$ & $0.016(2)$ & $0.013(2)$ & $0.028(2)$ \\
\hline $\mathrm{C} 22$ & $0.031(2)$ & $0.038(2)$ & $0.044(2)$ & $0.0097(17)$ & $0.0096(18)$ & $0.0064(18)$ \\
\hline
\end{tabular}

Geometric parameters $(\AA, \stackrel{o}{)})$

\begin{tabular}{llll}
\hline $\mathrm{C} 11-\mathrm{C} 4$ & $1.739(3)$ & $\mathrm{C} 11-\mathrm{C} 15$ & $1.485(6)$ \\
$\mathrm{S} 1-\mathrm{O} 1$ & $1.432(3)$ & $\mathrm{C} 11-\mathrm{C} 12$ & $1.551(6)$ \\
$\mathrm{S} 1-\mathrm{O} 2$ & $1.434(3)$ & $\mathrm{C} 11-\mathrm{H} 11$ & 1.0000
\end{tabular}




\begin{tabular}{|c|c|c|c|}
\hline $\mathrm{S} 1-\mathrm{C} 1$ & $1.753(4)$ & $\mathrm{C} 12-\mathrm{C} 13$ & $1.607(7)$ \\
\hline $\mathrm{S} 1-\mathrm{C} 2$ & $1.774(3)$ & $\mathrm{C} 12-\mathrm{H} 12 \mathrm{~A}$ & 0.9900 \\
\hline $\mathrm{S} 2-\mathrm{C} 10$ & $1.742(4)$ & $\mathrm{C} 12-\mathrm{H} 12 \mathrm{~B}$ & 0.9900 \\
\hline $\mathrm{S} 2-\mathrm{C} 17$ & $1.790(4)$ & $\mathrm{C} 13-\mathrm{C} 14$ & $1.425(8)$ \\
\hline $\mathrm{O} 3-\mathrm{C} 8$ & $1.213(4)$ & $\mathrm{C} 13-\mathrm{C} 16$ & $1.534(6)$ \\
\hline $\mathrm{O} 4-\mathrm{C} 16$ & $1.226(4)$ & $\mathrm{C} 13-\mathrm{H} 13$ & 1.0000 \\
\hline $\mathrm{C} 1-\mathrm{H} 1 \mathrm{~A}$ & 0.9800 & $\mathrm{C} 14-\mathrm{C} 15$ & $1.437(7)$ \\
\hline $\mathrm{C} 1-\mathrm{H} 1 \mathrm{~B}$ & 0.9800 & $\mathrm{C} 14-\mathrm{H} 14 \mathrm{~A}$ & 0.9900 \\
\hline $\mathrm{C} 1-\mathrm{H} 1 \mathrm{C}$ & 0.9800 & C14-H14B & 0.9900 \\
\hline $\mathrm{C} 2-\mathrm{C} 7$ & $1.385(5)$ & $\mathrm{C} 15-\mathrm{H} 15 \mathrm{~A}$ & 0.9900 \\
\hline $\mathrm{C} 2-\mathrm{C} 3$ & $1.386(5)$ & C15-H15B & 0.9900 \\
\hline $\mathrm{C} 3-\mathrm{C} 4$ & $1.384(5)$ & $\mathrm{C} 17-\mathrm{C} 18$ & $1.382(5)$ \\
\hline $\mathrm{C} 3-\mathrm{H} 3$ & 0.9500 & $\mathrm{C} 17-\mathrm{C} 22$ & $1.385(5)$ \\
\hline $\mathrm{C} 4-\mathrm{C} 5$ & $1.385(5)$ & $\mathrm{C} 18-\mathrm{C} 19$ & $1.383(6)$ \\
\hline $\mathrm{C} 5-\mathrm{C} 6$ & $1.395(5)$ & $\mathrm{C} 18-\mathrm{H} 18$ & 0.9500 \\
\hline $\mathrm{C} 5-\mathrm{C} 8$ & $1.508(5)$ & $\mathrm{C} 19-\mathrm{C} 20$ & $1.387(6)$ \\
\hline $\mathrm{C} 6-\mathrm{C} 7$ & $1.384(5)$ & C19-H19 & 0.9500 \\
\hline C6- 6 6 & 0.9500 & $\mathrm{C} 20-\mathrm{C} 21$ & $1.365(7)$ \\
\hline $\mathrm{C} 7-\mathrm{H} 7$ & 0.9500 & $\mathrm{C} 20-\mathrm{H} 20$ & 0.9500 \\
\hline $\mathrm{C} 8-\mathrm{C} 9$ & $1.487(5)$ & $\mathrm{C} 21-\mathrm{C} 22$ & $1.383(6)$ \\
\hline $\mathrm{C} 9-\mathrm{C} 10$ & $1.363(5)$ & $\mathrm{C} 21-\mathrm{H} 21$ & 0.9500 \\
\hline $\mathrm{C} 9-\mathrm{C} 16$ & $1.476(5)$ & $\mathrm{C} 22-\mathrm{H} 22$ & 0.9500 \\
\hline $\mathrm{C} 10-\mathrm{C} 11$ & $1.517(5)$ & & \\
\hline $\mathrm{O} 1-\mathrm{S} 1-\mathrm{O} 2$ & $119.16(18)$ & $\mathrm{C} 11-\mathrm{C} 12-\mathrm{C} 13$ & $95.2(3)$ \\
\hline $\mathrm{O} 1-\mathrm{S} 1-\mathrm{C} 1$ & $108.84(18)$ & $\mathrm{C} 11-\mathrm{C} 12-\mathrm{H} 12 \mathrm{~A}$ & 112.7 \\
\hline $\mathrm{O} 2-\mathrm{S} 1-\mathrm{C} 1$ & $108.9(2)$ & $\mathrm{C} 13-\mathrm{C} 12-\mathrm{H} 12 \mathrm{~A}$ & 112.7 \\
\hline $\mathrm{O} 1-\mathrm{S} 1-\mathrm{C} 2$ & $107.89(18)$ & $\mathrm{C} 11-\mathrm{C} 12-\mathrm{H} 12 \mathrm{~B}$ & 112.7 \\
\hline $\mathrm{O} 2-\mathrm{S} 1-\mathrm{C} 2$ & $108.04(16)$ & $\mathrm{C} 13-\mathrm{C} 12-\mathrm{H} 12 \mathrm{~B}$ & 112.7 \\
\hline $\mathrm{C} 1-\mathrm{S} 1-\mathrm{C} 2$ & $102.72(17)$ & $\mathrm{H} 12 \mathrm{~A}-\mathrm{C} 12-\mathrm{H} 12 \mathrm{~B}$ & 110.2 \\
\hline $\mathrm{C} 10-\mathrm{S} 2-\mathrm{C} 17$ & $103.94(16)$ & $\mathrm{C} 14-\mathrm{C} 13-\mathrm{C} 16$ & $105.7(5)$ \\
\hline $\mathrm{S} 1-\mathrm{C} 1-\mathrm{H} 1 \mathrm{~A}$ & 109.5 & $\mathrm{C} 14-\mathrm{C} 13-\mathrm{C} 12$ & $102.8(4)$ \\
\hline $\mathrm{S} 1-\mathrm{C} 1-\mathrm{H} 1 \mathrm{~B}$ & 109.5 & $\mathrm{C} 16-\mathrm{C} 13-\mathrm{C} 12$ & $111.0(3)$ \\
\hline $\mathrm{H} 1 \mathrm{~A}-\mathrm{C} 1-\mathrm{H} 1 \mathrm{~B}$ & 109.5 & $\mathrm{C} 14-\mathrm{C} 13-\mathrm{H} 13$ & 112.2 \\
\hline $\mathrm{S} 1-\mathrm{C} 1-\mathrm{H} 1 \mathrm{C}$ & 109.5 & $\mathrm{C} 16-\mathrm{C} 13-\mathrm{H} 13$ & 112.2 \\
\hline $\mathrm{H} 1 \mathrm{~A}-\mathrm{C} 1-\mathrm{H} 1 \mathrm{C}$ & 109.5 & $\mathrm{C} 12-\mathrm{C} 13-\mathrm{H} 13$ & 112.2 \\
\hline $\mathrm{H} 1 \mathrm{~B}-\mathrm{C} 1-\mathrm{H} 1 \mathrm{C}$ & 109.5 & $\mathrm{C} 13-\mathrm{C} 14-\mathrm{C} 15$ & $108.8(4)$ \\
\hline $\mathrm{C} 7-\mathrm{C} 2-\mathrm{C} 3$ & $121.3(3)$ & $\mathrm{C} 13-\mathrm{C} 14-\mathrm{H} 14 \mathrm{~A}$ & 109.9 \\
\hline $\mathrm{C} 7-\mathrm{C} 2-\mathrm{S} 1$ & $120.4(3)$ & $\mathrm{C} 15-\mathrm{C} 14-\mathrm{H} 14 \mathrm{~A}$ & 109.9 \\
\hline $\mathrm{C} 3-\mathrm{C} 2-\mathrm{S} 1$ & $118.3(3)$ & $\mathrm{C} 13-\mathrm{C} 14-\mathrm{H} 14 \mathrm{~B}$ & 109.9 \\
\hline $\mathrm{C} 4-\mathrm{C} 3-\mathrm{C} 2$ & $118.4(3)$ & $\mathrm{C} 15-\mathrm{C} 14-\mathrm{H} 14 \mathrm{~B}$ & 109.9 \\
\hline $\mathrm{C} 4-\mathrm{C} 3-\mathrm{H} 3$ & 120.8 & $\mathrm{H} 14 \mathrm{~A}-\mathrm{C} 14-\mathrm{H} 14 \mathrm{~B}$ & 108.3 \\
\hline $\mathrm{C} 2-\mathrm{C} 3-\mathrm{H} 3$ & 120.8 & $\mathrm{C} 14-\mathrm{C} 15-\mathrm{C} 11$ & $107.1(4)$ \\
\hline $\mathrm{C} 3-\mathrm{C} 4-\mathrm{C} 5$ & $121.9(3)$ & $\mathrm{C} 14-\mathrm{C} 15-\mathrm{H} 15 \mathrm{~A}$ & 110.3 \\
\hline $\mathrm{C} 3-\mathrm{C} 4-\mathrm{Cl} 1$ & $116.1(3)$ & $\mathrm{C} 11-\mathrm{C} 15-\mathrm{H} 15 \mathrm{~A}$ & 110.3 \\
\hline $\mathrm{C} 5-\mathrm{C} 4-\mathrm{Cl1}$ & $121.8(3)$ & $\mathrm{C} 14-\mathrm{C} 15-\mathrm{H} 15 \mathrm{~B}$ & 110.3 \\
\hline $\mathrm{C} 4-\mathrm{C} 5-\mathrm{C} 6$ & $118.3(3)$ & $\mathrm{C} 11-\mathrm{C} 15-\mathrm{H} 15 \mathrm{~B}$ & 110.3 \\
\hline $\mathrm{C} 4-\mathrm{C} 5-\mathrm{C} 8$ & $124.4(3)$ & $\mathrm{H} 15 \mathrm{~A}-\mathrm{C} 15-\mathrm{H} 15 \mathrm{~B}$ & 108.6 \\
\hline
\end{tabular}




\begin{tabular}{|c|c|c|c|}
\hline $\mathrm{C} 6-\mathrm{C} 5-\mathrm{C} 8$ & $117.2(3)$ & $\mathrm{O} 4-\mathrm{C} 16-\mathrm{C} 9$ & $121.8(3)$ \\
\hline $\mathrm{C} 7-\mathrm{C} 6-\mathrm{C} 5$ & $120.9(3)$ & $\mathrm{O} 4-\mathrm{C} 16-\mathrm{C} 13$ & $121.9(4)$ \\
\hline $\mathrm{C} 7-\mathrm{C} 6-\mathrm{H} 6$ & 119.5 & $\mathrm{C} 9-\mathrm{C} 16-\mathrm{C} 13$ & $116.1(3)$ \\
\hline $\mathrm{C} 5-\mathrm{C} 6-\mathrm{H} 6$ & 119.5 & $\mathrm{C} 18-\mathrm{C} 17-\mathrm{C} 22$ & $120.2(3)$ \\
\hline $\mathrm{C} 6-\mathrm{C} 7-\mathrm{C} 2$ & $119.1(3)$ & $\mathrm{C} 18-\mathrm{C} 17-\mathrm{S} 2$ & $120.6(3)$ \\
\hline $\mathrm{C} 6-\mathrm{C} 7-\mathrm{H} 7$ & 120.4 & $\mathrm{C} 22-\mathrm{C} 17-\mathrm{S} 2$ & $119.0(3)$ \\
\hline $\mathrm{C} 2-\mathrm{C} 7-\mathrm{H} 7$ & 120.4 & $\mathrm{C} 17-\mathrm{C} 18-\mathrm{C} 19$ & $120.1(4)$ \\
\hline $\mathrm{O} 3-\mathrm{C} 8-\mathrm{C} 9$ & $120.9(3)$ & $\mathrm{C} 17-\mathrm{C} 18-\mathrm{H} 18$ & 119.9 \\
\hline $\mathrm{O} 3-\mathrm{C} 8-\mathrm{C} 5$ & $118.2(3)$ & $\mathrm{C} 19-\mathrm{C} 18-\mathrm{H} 18$ & 119.9 \\
\hline $\mathrm{C} 9-\mathrm{C} 8-\mathrm{C} 5$ & $120.9(3)$ & $\mathrm{C} 18-\mathrm{C} 19-\mathrm{C} 20$ & $119.2(4)$ \\
\hline $\mathrm{C} 10-\mathrm{C} 9-\mathrm{C} 16$ & $119.7(3)$ & $\mathrm{C} 18-\mathrm{C} 19-\mathrm{H} 19$ & 120.4 \\
\hline $\mathrm{C} 10-\mathrm{C} 9-\mathrm{C} 8$ & $120.7(3)$ & $\mathrm{C} 20-\mathrm{C} 19-\mathrm{H} 19$ & 120.4 \\
\hline $\mathrm{C} 16-\mathrm{C} 9-\mathrm{C} 8$ & $119.6(3)$ & $\mathrm{C} 21-\mathrm{C} 20-\mathrm{C} 19$ & $120.8(4)$ \\
\hline $\mathrm{C} 9-\mathrm{C} 10-\mathrm{C} 11$ & $119.4(3)$ & $\mathrm{C} 21-\mathrm{C} 20-\mathrm{H} 20$ & 119.6 \\
\hline $\mathrm{C} 9-\mathrm{C} 10-\mathrm{S} 2$ & $121.9(3)$ & $\mathrm{C} 19-\mathrm{C} 20-\mathrm{H} 20$ & 119.6 \\
\hline $\mathrm{C} 11-\mathrm{C} 10-\mathrm{S} 2$ & $118.7(3)$ & $\mathrm{C} 20-\mathrm{C} 21-\mathrm{C} 22$ & $120.3(4)$ \\
\hline $\mathrm{C} 15-\mathrm{C} 11-\mathrm{C} 10$ & $110.0(3)$ & $\mathrm{C} 20-\mathrm{C} 21-\mathrm{H} 21$ & 119.9 \\
\hline $\mathrm{C} 15-\mathrm{C} 11-\mathrm{C} 12$ & $103.2(4)$ & $\mathrm{C} 22-\mathrm{C} 21-\mathrm{H} 21$ & 119.9 \\
\hline $\mathrm{C} 10-\mathrm{C} 11-\mathrm{C} 12$ & $107.9(3)$ & $\mathrm{C} 21-\mathrm{C} 22-\mathrm{C} 17$ & $119.5(4)$ \\
\hline $\mathrm{C} 15-\mathrm{C} 11-\mathrm{H} 11$ & 111.8 & $\mathrm{C} 21-\mathrm{C} 22-\mathrm{H} 22$ & 120.3 \\
\hline $\mathrm{C} 10-\mathrm{C} 11-\mathrm{H} 11$ & 111.8 & $\mathrm{C} 17-\mathrm{C} 22-\mathrm{H} 22$ & 120.3 \\
\hline $\mathrm{C} 12-\mathrm{C} 11-\mathrm{H} 11$ & 111.8 & & \\
\hline $\mathrm{O} 1-\mathrm{S} 1-\mathrm{C} 2-\mathrm{C} 7$ & $-37.9(4)$ & $\mathrm{C} 17-\mathrm{S} 2-\mathrm{C} 10-\mathrm{C} 11$ & $19.8(3)$ \\
\hline $\mathrm{O} 2-\mathrm{S} 1-\mathrm{C} 2-\mathrm{C} 7$ & $-167.9(3)$ & $\mathrm{C} 9-\mathrm{C} 10-\mathrm{C} 11-\mathrm{C} 15$ & $64.1(5)$ \\
\hline $\mathrm{C} 1-\mathrm{S} 1-\mathrm{C} 2-\mathrm{C} 7$ & $77.0(3)$ & $\mathrm{S} 2-\mathrm{C} 10-\mathrm{C} 11-\mathrm{C} 15$ & $-116.0(4)$ \\
\hline $\mathrm{O} 1-\mathrm{S} 1-\mathrm{C} 2-\mathrm{C} 3$ & $145.0(3)$ & $\mathrm{C} 9-\mathrm{C} 10-\mathrm{C} 11-\mathrm{C} 12$ & $-47.8(5)$ \\
\hline $\mathrm{O} 2-\mathrm{S} 1-\mathrm{C} 2-\mathrm{C} 3$ & $14.9(4)$ & $\mathrm{S} 2-\mathrm{C} 10-\mathrm{C} 11-\mathrm{C} 12$ & $132.1(3)$ \\
\hline $\mathrm{C} 1-\mathrm{S} 1-\mathrm{C} 2-\mathrm{C} 3$ & $-100.1(3)$ & $\mathrm{C} 15-\mathrm{C} 11-\mathrm{C} 12-\mathrm{C} 13$ & $-42.9(4)$ \\
\hline $\mathrm{C} 7-\mathrm{C} 2-\mathrm{C} 3-\mathrm{C} 4$ & $0.8(6)$ & $\mathrm{C} 10-\mathrm{C} 11-\mathrm{C} 12-\mathrm{C} 13$ & $73.5(4)$ \\
\hline $\mathrm{S} 1-\mathrm{C} 2-\mathrm{C} 3-\mathrm{C} 4$ & $177.9(3)$ & $\mathrm{C} 11-\mathrm{C} 12-\mathrm{C} 13-\mathrm{C} 14$ & $44.6(4)$ \\
\hline $\mathrm{C} 2-\mathrm{C} 3-\mathrm{C} 4-\mathrm{C} 5$ & $-1.5(6)$ & $\mathrm{C} 11-\mathrm{C} 12-\mathrm{C} 13-\mathrm{C} 16$ & $-68.0(5)$ \\
\hline $\mathrm{C} 2-\mathrm{C} 3-\mathrm{C} 4-\mathrm{Cl} 1$ & $-178.0(3)$ & $\mathrm{C} 16-\mathrm{C} 13-\mathrm{C} 14-\mathrm{C} 15$ & $86.1(5)$ \\
\hline $\mathrm{C} 3-\mathrm{C} 4-\mathrm{C} 5-\mathrm{C} 6$ & $0.9(6)$ & $\mathrm{C} 12-\mathrm{C} 13-\mathrm{C} 14-\mathrm{C} 15$ & $-30.4(5)$ \\
\hline $\mathrm{C} 11-\mathrm{C} 4-\mathrm{C} 5-\mathrm{C} 6$ & $177.3(3)$ & $\mathrm{C} 13-\mathrm{C} 14-\mathrm{C} 15-\mathrm{C} 11$ & $2.1(5)$ \\
\hline $\mathrm{C} 3-\mathrm{C} 4-\mathrm{C} 5-\mathrm{C} 8$ & $-174.6(3)$ & $\mathrm{C} 10-\mathrm{C} 11-\mathrm{C} 15-\mathrm{C} 14$ & $-86.7(4)$ \\
\hline $\mathrm{C} 11-\mathrm{C} 4-\mathrm{C} 5-\mathrm{C} 8$ & $1.8(5)$ & $\mathrm{C} 12-\mathrm{C} 11-\mathrm{C} 15-\mathrm{C} 14$ & $28.3(4)$ \\
\hline $\mathrm{C} 4-\mathrm{C} 5-\mathrm{C} 6-\mathrm{C} 7$ & $0.4(6)$ & $\mathrm{C} 10-\mathrm{C} 9-\mathrm{C} 16-\mathrm{O} 4$ & $177.3(4)$ \\
\hline $\mathrm{C} 8-\mathrm{C} 5-\mathrm{C} 6-\mathrm{C} 7$ & $176.2(3)$ & $\mathrm{C} 8-\mathrm{C} 9-\mathrm{C} 16-\mathrm{O} 4$ & $-1.1(7)$ \\
\hline $\mathrm{C} 5-\mathrm{C} 6-\mathrm{C} 7-\mathrm{C} 2$ & $-1.0(6)$ & $\mathrm{C} 10-\mathrm{C} 9-\mathrm{C} 16-\mathrm{C} 13$ & $0.7(6)$ \\
\hline $\mathrm{C} 3-\mathrm{C} 2-\mathrm{C} 7-\mathrm{C} 6$ & $0.5(6)$ & $\mathrm{C} 8-\mathrm{C} 9-\mathrm{C} 16-\mathrm{C} 13$ & $-177.6(4)$ \\
\hline $\mathrm{S} 1-\mathrm{C} 2-\mathrm{C} 7-\mathrm{C} 6$ & $-176.6(3)$ & $\mathrm{C} 14-\mathrm{C} 13-\mathrm{C} 16-\mathrm{O} 4$ & $106.6(5)$ \\
\hline $\mathrm{C} 4-\mathrm{C} 5-\mathrm{C} 8-\mathrm{O} 3$ & $131.3(4)$ & $\mathrm{C} 12-\mathrm{C} 13-\mathrm{C} 16-\mathrm{O} 4$ & $-142.6(5)$ \\
\hline $\mathrm{C} 6-\mathrm{C} 5-\mathrm{C} 8-\mathrm{O} 3$ & $-44.2(5)$ & $\mathrm{C} 14-\mathrm{C} 13-\mathrm{C} 16-\mathrm{C} 9$ & $-76.9(5)$ \\
\hline $\mathrm{C} 4-\mathrm{C} 5-\mathrm{C} 8-\mathrm{C} 9$ & $-46.5(5)$ & $\mathrm{C} 12-\mathrm{C} 13-\mathrm{C} 16-\mathrm{C} 9$ & $33.9(6)$ \\
\hline $\mathrm{C} 6-\mathrm{C} 5-\mathrm{C} 8-\mathrm{C} 9$ & $138.0(4)$ & $\mathrm{C} 10-\mathrm{S} 2-\mathrm{C} 17-\mathrm{C} 18$ & $74.3(3)$ \\
\hline $\mathrm{O} 3-\mathrm{C} 8-\mathrm{C} 9-\mathrm{C} 10$ & $-31.3(6)$ & $\mathrm{C} 10-\mathrm{S} 2-\mathrm{C} 17-\mathrm{C} 22$ & $-111.6(3)$ \\
\hline $\mathrm{C} 5-\mathrm{C} 8-\mathrm{C} 9-\mathrm{C} 10$ & $146.4(4)$ & $\mathrm{C} 22-\mathrm{C} 17-\mathrm{C} 18-\mathrm{C} 19$ & $0.5(6)$ \\
\hline
\end{tabular}




$\begin{array}{llll}\mathrm{O} 3-\mathrm{C} 8-\mathrm{C} 9-\mathrm{C} 16 & 147.0(4) & \mathrm{S} 2-\mathrm{C} 17-\mathrm{C} 18-\mathrm{C} 19 & 174.5(3) \\ \mathrm{C} 5-\mathrm{C} 8-\mathrm{C} 9-\mathrm{C} 16 & -35.3(5) & \mathrm{C} 17-\mathrm{C} 18-\mathrm{C} 19-\mathrm{C} 20 & -1.0(6) \\ \mathrm{C} 16-\mathrm{C} 9-\mathrm{C} 10-\mathrm{C} 11 & 5.7(6) & \mathrm{C} 18-\mathrm{C} 19-\mathrm{C} 20-\mathrm{C} 21 & 1.0(7) \\ \mathrm{C} 8-\mathrm{C} 9-\mathrm{C} 10-\mathrm{C} 11 & -176.0(3) & \mathrm{C} 19-\mathrm{C} 20-\mathrm{C} 21-\mathrm{C} 22 & -0.4(6) \\ \mathrm{C} 16-\mathrm{C} 9-\mathrm{C} 10-\mathrm{S} 2 & -174.2(3) & \mathrm{C} 20-\mathrm{C} 21-\mathrm{C} 22-\mathrm{C} 17 & -0.1(6) \\ \mathrm{C} 8-\mathrm{C} 9-\mathrm{C} 10-\mathrm{S} 2 & 4.1(5) & \mathrm{C} 18-\mathrm{C} 17-\mathrm{C} 22-\mathrm{C} 21 & 0.1(5) \\ \mathrm{C} 17-\mathrm{S} 2-\mathrm{C} 10-\mathrm{C} 9 & -160.3(3) & \mathrm{S} 2-\mathrm{C} 17-\mathrm{C} 22-\mathrm{C} 21 & -174.0(3)\end{array}$

Hydrogen-bond geometry $\left(A,{ }^{\circ}\right)$

\begin{tabular}{lllll}
\hline$D-\mathrm{H} \cdots A$ & $D-\mathrm{H}$ & $\mathrm{H} \cdots A$ & $D \cdots A$ & $D-\mathrm{H}^{\cdots} A$ \\
\hline $\mathrm{C} 1-\mathrm{H} 1 B \cdots \mathrm{O} 4^{\mathrm{i}}$ & 0.98 & 2.44 & $3.322(4)$ & 150 \\
$\mathrm{C} 1-\mathrm{H} 1 C \cdots \mathrm{O} 4^{\mathrm{ii}}$ & 0.98 & 2.52 & $3.477(5)$ & 165 \\
$\mathrm{C} 3-\mathrm{H} 3 \cdots \mathrm{O} 2^{\mathrm{iii}}$ & 0.95 & 2.53 & $3.422(4)$ & 157 \\
\hline
\end{tabular}

Symmetry codes: (i) $x,-y+1 / 2, z+1 / 2$; (ii) $-x, y-1 / 2,-z+3 / 2$; (iii) $-x,-y+1,-z+2$. 